\title{
La competencia comunicativa intercultural en la clase multicultural de español como lengua extranjera
}

\author{
Esther Diviñó-González ${ }^{\star}$
}

* M.Sc. en Linguodidáctica. Docente, Facultad de Lenguas Extranjeras, Universidad de La Habana, Cuba.

Correo electrónico:

esther.divino@rect.uh.cu

Recibido: 28 de agosto del 2013 Aprobado: 7 de octubre del 2013

Cómo citar este artículo: Diviñó-González, Esther. "La competencia comunicativa intercultural en la clase multicultural de español como lengua extranjera". Rastros Rostros 15.29 (2013): 25-35. Impreso.

\begin{abstract}
Resumen
En el presente artículo nos proponemos reflexionar acerca del desafío a que estamos enfrentados como resultado del uso inapropiado que se hace, en no pocas ocasiones, de la ciencia, la tecnología y las comunicaciones, por lo que es necesario desarrollar la competencia comunicativa intercultural y clarificar valores en grupos multiculturales de estudiantes que aprenden español como lengua extranjera (E/LE) fuera de su país. La investigación que origina este artículo corresponde a la tesis doctoral El tratamiento de los valores en condiciones de interculturalidad a partir de la comprensión lectora: estrategia linguodidáctica. En la metodología, la autora empleó el modelo investigación en la acción, que es propio de las disciplinas didácticas, e hizo un ensayo con un repertorio de textos tomados de la literatura cubana y latinoamericana, con el objetivo de trabajarlos en el nivel superior de la enseñanza de E/LE. A partir de los propios textos y del sistema de tareas que de ellos se derivan, dentro de las que no faltan actividades referidas a las diferencias culturales y a los valores, se pudo comprobar cómo la mayoría de los estudiantes alcanza la competencia intercultural, al tiempo que comprende mejor los valores "del otro" y amplía su horizonte apreciativo.
\end{abstract}

Palabras clave: competencia intercultural, conflictos, cultura, multiculturalidad, textos, valores.

Intercultural Communicative Skills in the Multicultural Classroom for Spanish as a Foreign Language

\section{Abstract}

This article reflects on the challenge created by the inappropriate use, on many occasions, of science, technology and communication, making it necessary to develop intercultural communication skills and shine a light on values in multicultural groups of students studying Spanish as a foreign language (E/EL) outside their countries. The research in this article corresponds to the doctoral thesis Treatment of values in intercultural conditions based on reading comprehension: linguistic-didactic strategy. In this methodology, the author employed the action research model -germane to didactic disciplines- and ran a trial using a repertoire of texts taken from Cuban and Latin American literature, with the aim of working with these in advanced levels of E/EL teaching. Based on the texts themselves, and the tasks taken from them, which included activities that referred to differences in cultures and values, it was confirmed that the majority of students gain intercultural skills when they better understand the values of "the other" and widen their appreciative horizons.

Keywords: intercultural skills, conflicts, culture, multiculturality, texts, values.

\section{A competência comunicativa intercultural na aula multicultural de espanhol como língua estrangeira \\ Resumo}

No presente artigo nos propomos refletir acerca do desafio que estamos enfrentando, como resultado do uso inapropriado que se faz, não em poucas ocasiões, da ciência, da tecnologia e das comunicações, razão pela qual é necessário desenvolver a competência comunicativa intercultural e esclarecer valores em turmas multiculturais de estudantes que aprendem espanhol como língua estrangeira $(\mathrm{E} / \mathrm{LE})$ fora do país. A pesquisa que origina este artigo corresponde à tese de doutorado $O$ tratamento dos valores em condições de interculturalidade a partir da compreensão leitora: estratégia linguodidática. Na metodologia, a autora empregou o modelo de investigação-ação, que é próprio das disciplinas didáticas, e fez um ensaio com um repertório de textos retirados da literatura cubana e latino-americana, com o objetivo de serem trabalhados no nível superior de ensino de e/le. A partir dos próprios textos e do sistema de tarefas que deles se derivam, dentro das quais não faltam atividades referidas às diferenças culturais $e$ aos valores, pôde-se comprovar como a maioria dos estudantes alcança a competência intercultural, ao passo que compreende melhor os valores "do outro" e amplia seu horizonte apreciativo.

Palavras-chave: competência intercultural, conflitos, cultura, multiculturalidade, textos, valores. 


\section{Introducción}

Estemos o no de acuerdo, nos enfrentamos a un mundo nuevo y complejo en el que la ciencia y la tecnología están construyendo su propio universo de sentidos y están abriendo paso a una forma de cultura diferente. Estas transformaciones están acompañadas por cambios profundos que obligan a un nuevo nacimiento humano en el que parece desaparecer el tiempo y la distancia. Frente a ellos, no podemos aventurarnos a catalogar la nueva vida como mejor o peor que la anterior; pero sí, que hasta ahora no existe una conciencia clara de su gobernabilidad. Por tanto, los diagnósticos son aún muy reservados, sus efectos económicos y sociales todavía no pueden determinarse a escala global.

Sin embargo, "este hoy que puede ser el mañana", como dice Víctor Jara en una de sus canciones, está totalmente moldeado y dominado por la informática; nuevos y no previstos problemas creados por la ciencia y la tecnología multiplican los riesgos económicos, políticos y sociales. Mientras tanto, se va conformando un nuevo mundo y una nueva forma de cultura caracterizados por la inmediatez de los mensajes, la multiplicación del conocimiento y la diversidad mundial.

Como respuesta al reto a que nos conduce el uso, en ocasiones, inapropiado de la disponibilidad científico-técnica en la actual coyuntura internacional, la cual no pocas veces obstaculiza la formación de un ser humano íntegro, debemos mantener como principio la elevación continua de la calidad de la educación superior sobre bases científicas que incluyan la implementación y el perfeccionamiento de acciones pedagógicas de las cuales se deriven actividades de carácter ético y axiológico, como expresiones de un sentido integrador de la calidad del proceso docente.

No resulta difícil comprobar que el mundo actual se caracteriza por su gran diversidad; la multiculturalidad, como "situación social (nacional, regional, comunitaria, grupal) de culturas en contacto" (Trujillo 10), se intensifica también en nuestras aulas, y se hacen más necesarias y hasta imprescindibles las relaciones interculturales.

La enseñanza del español como lengua extranjera (E/LE) en el ámbito de la educación superior en La Universidad de la Habana acoge regularmente grupos de estudiantes que se caracterizan por su pluralidad cultural. Esta circunstancia dificulta enormemente el desarrollo de la competencia comunicativa intercultural en los términos en que viene propuesta por el Marco Co- mún Europeo de Referencia para las Lenguas (MCERL). Así, los grupos multiculturales de E/LE evidencian habitualmente actitudes conservadoras que resaltan la superioridad de ciertas culturas sobre otras, posiciones nacionalistas, cerradas, poco dispuestas al diálogo, o marcas discursivas con semántica valorativa axiológicamente negativa que caracterizan el discurso en la etapa etnocéntrica y que se expresan por medio de:

1. Caracterizaciones estereotipadas.

2. Muestra de aparente compasión y altruismo.

3. Comparación negativa.

4. Generalizaciones.

Por lo anteriormente expuesto, es objetivo del presente trabajo compartir los resultados alcanzados hasta este momento por una investigación que se propuso desarrollar la competencia comunicativa intercultural y clarificar valores en los grupos multiculturales de estudiantes que aprenden $\mathrm{E} / \mathrm{LE}$ fuera de su país de origen, específicamente, en este caso, en la Universidad de La Habana.

Para cumplimentar los objetivos propuestos se seleccionó un grupo de textos literarios para elaborar un repertorio que propiciara actividades integradas de comprensión lectora, expresión e interacción oral en grupos multiculturales de nivel superior.

\section{Desarrollo}

\section{Antecedentes y estado actual del tema}

La mayoría de los trabajos desarrollados en los últimos años para el tratamiento de conflictos interculturales hacen referencia a contextos sociales $-\mathrm{y}$ no necesariamente educativos. Entre las aportaciones teóricas conviene destacar la de Rocío Serrano, que plantea de forma crítica la posibilidad de distinguir "tres grandes políticas sociales y educativas con respecto al fenómeno multicultural en los países de nuestro entorno: asimilacionismo, segregacionismo e integracionismo". Nuestra investigación no persigue identificar los conflictos interculturales del aula con ninguna de estas tendencias, sino más bien mostrar que la diversidad cultural no es un obstáculo para la comunicación entre los participantes. Sí coincidimos con Rocío Serrano en la descripción de las condiciones mínimas que posibilitan un genuino interculturalismo, entre las que recoge: el reconocimiento de la diversidad, el derecho a la diferencia, la necesidad de construir lenguajes comu- 
nes y normas compartidas que permitan el diálogo, o el establecimiento negociado de fronteras entre códigos y normas comunes y específicas.

La profesora Teresa Aguado Odina (16), experta en diversidad cultural y educación, de la Universidad Nacional de Educación a Distancia, también ofrece un aporte teórico importante para este trabajo. Nos referimos al concepto de Pedagogía Intercultural, que viene desarrollando en los últimos años y que supone una incursión relevante en el terreno de la educación. Esta perspectiva señala que las diferencias culturales son la norma y están presentes en todo contexto educativo y social, por lo que se manifiesta en contra de las visiones restrictivas y marginalizadoras. El término intercultural, en opinión de la experta, implica intercambio e interacción entre diferentes marcos culturales.

Ya en el ámbito de la práctica educativa propiamente dicha recogemos algunos antecedentes importantes en la tesis doctoral Modelo didáctico para el tratamiento de la valoración apreciativa ético-psicológica en la clase de lengua extranjera, de Llanes Barbuzano, de la Universidad de La Habana, se propone un modelo didáctico para el tratamiento de los valores en la clase de E/LE. Su idea, sin embargo, se circunscribe a grupos de nivel elemental de español y se concibe para la valoración puramente semántica, no axiológica, como es nuestro caso. No obstante, deben reconocerse sus concepciones referidas a cómo se amplía el horizonte apreciativo del hombre al entrar en contacto con otras culturas y otros valores.

Dentro del ámbito de la práctica educativa conviene también rescatar un modelo didáctico que explicite suficientemente el papel del profesor de idiomas en las tareas dedicadas a la comprensión lectora. La tesis doctoral de Francisco Zayas Martínez (82-83), de la Universidad de Cádiz, España, El uso de la literatura en la enseñanza del alemán como segunda lengua extranjera, incluye, de hecho, propuestas y criterios para la selección de los textos en relación con el material lingüístico y el nivel de los estudiantes, el diseño de actividades relacionadas con la motivación y contextualización previo a la interacción texto-lector, así como procesos que facilitan la interacción y los procesos interculturales.

\section{Cultura para la interculturalidad}

La lengua es siempre espejo de la cultura y de las formas de vida de la colectividad que la habla. Es por tanto, en este sentido, algo susceptible al cambio, aunque con mucha más lentitud que cualquier otra manifestación cultural; se adapta con facilidad al entorno y nombra las relaciones sociales y culturales establecidas, de acuerdo con la época y sus diferentes usuarios, porque la lengua en sí no se presenta de forma única ni el individuo la posee en su totalidad.

La cultura posibilita comprender todas las circunstancias que puedan aparecer en la comunicación: dos o más individuos del mismo o de diferentes contextos culturales pueden dominar el mismo código lingüístico, pero si no poseen similares conocimientos socioculturales, difícilmente podrían compartir los mismos presupuestos y los mismos sobreentendidos, perderían parte de la comunicación o no podrían acceder a ella en toda su plenitud.

Las lenguas, entonces (y también las culturas), viven inmersas en las circunstancias sociales de las colectividades y son precisamente estas las que intervienen sobremanera en las formas de comunicarse. La lengua es el instrumento, con sus leyes propias, que la sociedad utiliza en cada momento histórico con todas sus posibilidades, y estas, a lo largo de los siglos varían: si la sociedad cambia — como es natural, al ser un ser vivo—, las formas que el lenguaje adopta para la comunicación se adaptan a lo nuevo, con el objetivo de que esta no se afecte.

La cultura se manifiesta en el lenguaje, en el pensamiento, en la manera en que los seres humanos deben comportarse en situaciones sociales concretas. De este modo, la gente va adaptándose a la conducta común $y$, en ocasiones, a las actitudes expresivas; por tanto, es aprendida, transmisible, dinámica y selectiva, $\mathrm{y}$, por supuesto, abarca todo aquello que el individuo debe saber para desenvolverse en una sociedad concreta. No debemos verla solamente como hiperónimo de manifestaciones artísticas que incluyen, al mismo tiempo, a la música, la pintura, la literatura, las artes plásticas, etcétera. Esto quiere decir que el término no debe ser entendido en un sentido restrictivo, ni selectivo, sino amplio.

Se hace imprescindible, entonces, contextualizar lo que significa para nosotros y para nuestras aulas el vocablo cultura, de modo que podamos elaborar un programa que conduzca a su desarrollo y constante evaluación. Existen diversas definiciones de este término, según las múltiples áreas y perspectivas de investigación e incluso dentro de la lingüística y la enseñanza de lenguas ha tenido y tiene diferentes enunciaciones. En el ensayo La enseñanza de idiomas en los últimos cien años: métodos y enfoques, de Aquilino Sánchez (308), se define así: 
Cultura es el conjunto de conocimientos, creencias, valores, actitudes y expresiones de la actividad o el quehacer de una sociedad o grupo social determinado. Normalmente los elementos que constituyen la cultura de un grupo social o pueblo pasan inadvertidos entre los miembros del mismo grupo. Pero algunos elementos, los que son distintivos, se ponen de relieve cuando dos o más grupos entran en contacto.

En este concepto se analiza que, de cierto modo, cultura es todo; lo cual incluye, implícitamente, educación y comunicación; pero además, se reconoce cómo destacan los elementos culturales cuando dos o más grupos sociales entran en contacto.

Conviene aquí precisar que la competencia comunicativa descansa sobre varios pilares; la lengua es uno de ellos -quizás el principal- y la cultura es otro de singular importancia. Cada uno de ellos es eficaz en su propia dimensión, pues contribuye a la eficacia comunicativa en conjunción con otros que configuran el proceso.

Puede decirse que en la clase de E/LE el componente cultural ha sido durante un tiempo el telón de fondo de los sistemas gramaticales y léxicos de la lengua. En el mejor de los casos, los profesores se han dedicado a transmitir informaciones sobre elementos culturales, pero no a reflexionar o a facilitar la comprensión de esos elementos. Tal postura, de alguna manera, ha contribuido a que los estudiantes no sean conscientes de las múltiples facetas de la identidad cultural (formas de pensar, actitudes, costumbres, valores, etc.) de la lengua que estudian.

En los últimos tiempos, lingüistas y profesores de idiomas comparten la concepción de que la enseñanza de lenguas es un proceso de interacción y comunicación social, como compartir el mismo significado referencial de las palabras. El artículo de Claire Kramsch (217), Contexto y cultura en la enseñanza de lenguas, indica cómo la conciencia cultural puede ayudar al desarrollo de la competencia comunicativa en la lengua extranjera, pues en el proceso de enseñanza-aprendizaje la cultura penetra, fruto de la interacción social, tanto en el habla como en la escritura.

Durante décadas nos hemos planteado como objetivo que los aprendices se acerquen lo más posible a la competencia lingüística de un hablante nativo, pero es fácil identificar comportamientos lingüísticos poco apropiados por parte de esos mismos hablantes no nativos. Sin embargo, al introducir el término cultura el objetivo de la enseñanza no va a ser que el estudiante se asemeje al nativo, sino que siendo consciente de su propia identidad y cultura, de cómo se le percibe, conozca las identidades y las culturas de las personas con las que interacciona.

A partir de esta concepción, se debe tener presente la formación multicultural, con toda la diversidad que esta implica, que tienen los grupos de E/LE al entrar en relación. En el artículo Cuando chocan las culturas (Richard D. Lewis citado en Diviñó, El desarrollo) se hace una caracterización general de tres tipos fundamentales, en los cuales incluyo algunos países que pueden ser representativos de cada tipo, según mi propia experiencia docente:

Las culturas multiactivas, las cuales define sucintamente como aquellas formadas por personas vivas, locuaces, de percepción temporal policromática (que realizan varias tareas a la vez), personas que en la planificación no priorizan las actividades de acuerdo con una agenda preestablecida, sino más bien de acuerdo con la importancia o gratificación de la actividad.

En este grupo se encuentran muchos de los países de Latinoamérica, incluyendo a Cuba, Brasil, Venezuela, Colombia, etc.

Las culturas reactivas, que son las que priorizan la cortesía y el respeto, saben escuchar con autocontrol a su interlocutor y reaccionan con mucho tacto ante la propuesta "del otro".

Aquí podemos encontrar a Japón y China, entre otros.

Las culturas activas lineales, las cuales planifican, siguen la organización de una agenda, están orientadas hacia el cumplimiento de las tareas y tienen una percepción temporal monocromática; es decir, realizan una sola tarea en un espacio de tiempo.

Entre los países de este grupo podemos citar a Inglaterra, Alemania, Francia y otros.

En la siguiente tabla proponemos una caracterización, según la clasificación de Lewis, para analizar cómo se reflejan dos de las oposiciones valorales que se observan claramente en diferentes culturas: 
Tabla 1. "Caracterización cultural y oposiciones valorales". Resume el análisis realizado por Richard D. Lewis en su artículo "Cuando chocan las culturas"

\begin{tabular}{|l|l|l|l|}
\hline & \multicolumn{3}{|c|}{ Clasificación de culturas } \\
\hline Oposiciones valorales & Activas lineales & Multiactivas & Reactivas \\
\hline $\begin{array}{l}\text { Cooperativismo - } \\
\text { competitividad }\end{array}$ & $\begin{array}{l}\text {-Rechazan aceptar favores; su } \\
\text { orientación básica es el trabajo y } \\
\text { no las relaciones sociales. }\end{array}$ & $\begin{array}{l}\text {-Son compasivos y rechazan apro- } \\
\text { vecharse de las debilidades ajenas. } \\
\text {-Además, muestran sus propias } \\
\text { debilidades. } \\
\text {-Están más orientados hacia las } \\
\text { personas que hacia el trabajo. }\end{array}$ & $\begin{array}{l}\text {-Son corteses, sacrificados, siem- } \\
\text { pre están prestos y dispuestos a la } \\
\text { cooperación recíproca. } \\
\text {-Están más orientados hacia las } \\
\text { personas que hacia el trabajo. }\end{array}$ \\
\hline Individualismo -colectivismo & $\begin{array}{l}\text {-Sociedad solo preocupada por } \\
\text { sus problemas y por defender su } \\
\text { privacidad. }\end{array}$ & $\begin{array}{l}\text {-Son gregarias (les gusta estar } \\
\text { en grupos), inquisitivas, buscan } \\
\text { influencias y piden favores. }\end{array}$ & $\begin{array}{l}\text {-Son respetuosas de la privacidad, } \\
\text { pero saben escuchar, crean redes } \\
\text { de ayuda y evitan crearles proble- } \\
\text { mas a sus semejantes. }\end{array}$ \\
\hline
\end{tabular}

Fuente. Diviño (El desarrollo 34).

Estos tres conjuntos de rasgos entran en relación en las clases de E/LE, lo que da lugar a los desacuerdos antes mencionados y limitan el progreso de la competencia comunicativa intercultural. Resulta aconsejable y provechoso que el profesor conozca las características de cada una de las culturas presentes en su clase.

¿Qué significa interculturalidad en las aulas de E/LE?

La interculturalidad se puede describir en términos estáticos y dinámicos: se describe estáticamente cuando se utiliza para hablar de una situación comunicativa en la que se ponen en contacto dos o más individuos que se perciben el uno al otro como pertenecientes a distintas culturas; se describe dinámicamente, cuando se utiliza para hablar de los mecanismos y procesos que se ponen en funcionamiento en esa interacción comunicativa y, especialmente, para que esa comunicación sea efectiva. (Trujillo 11)

En general, como plantea Trujillo, el término interculturalidad hace referencia al encuentro entre personas que provienen de entornos socioculturales $y$ sociolingüísticos distintos, en los que han operado procesos a través de los cuales se les han ido formando valores, creencias, conocimientos y formas de pensar, hablar, sentir y actuar propias de su medio sociocultural. Pero este tipo de encuentro puede ser de muchas clases: improvisado, casual, formalizado; adopta muchas caras, propias de la vida moderna, con su movilidad y sus cruces constantes; gracias a ellas las sociedades se exponen a nuevos ambientes, se aprenden y se transmiten nuevos comportamientos, prácticas, ideas, conocimientos, valores, creencias y actitudes culturales. Sin embargo, este artículo intenta hacer referencia a una interculturalidad más explícita, propia de entornos educativos donde la enseñanza y la formación ne- cesitan estrategias pedagógicas reguladas, así como prácticas comunicativas e interactivas determinadas y consensuadas dentro del proceso de aprendizaje. Puede decirse que educar para la interculturalidad es, pedagógicamente hablando, una tarea fundamentalmente lingüístico-comunicativa y cognitivo-axiológica, es decir, de comunicación y comprensión de valores.

La interculturalidad supone, además, respeto a la diversidad; no niega la obra citada, la aparición de conflictos, incluso aclara que son inevitables; pero estos se resuelven con respeto, diálogo y concertación; analiza cómo se facilita el "encuentro de dos o más culturas cuando entran en interacción de una forma horizontal y sinérgica", lo cual no significa que desaparezcan las relaciones de poder, pero sí que puedan matizarse, equilibrarse, y así favorecer la interacción y la convivencia de las personas.

Sobre la base de las afirmaciones anteriores puede decirse que, en la clase, las relaciones interculturales se consiguen mediante tres condiciones: una visión dinámica de las culturas, una comunicación basada en la comprensión de la cultura "del otro" y el logro de relaciones equilibradas de poder.

Según los análisis de Trujillo, la competencia intercultural debe desarrollarse durante el proceso de aprendizaje de una lengua extranjera (LE), en el que participen estudiantes de diferentes culturas, puesto que en ella se expresan valores universales a partir del conocimiento de la propia cultura y de la comprensión de las demás.

Sin embargo, el logro de la interculturalidad o de las relaciones interculturales en el aula de LE no es sencillo, ni podemos verlo de manera aislada, porque forma parte de un concepto más abarcador, que es el de relación. En el proceso de enseñanza aprendizaje de E $\mid$ LE se habla 
de relación, porque entre los elementos que constituyen este proceso (profesores, estudiantes, métodos, materiales) se establece un vínculo con el mundo exterior y el mundo interior "del otro". Este vínculo se materializa en la tarea de enseñar y aprender a comunicarse con, por lo menos, dos mundos culturales que entran en contacto: el de los estudiantes que aprenden fuera de su país de origen y el del profesor que enseña en el medio lingüístico.

En el mundo de la clase de E $\backslash_{\mathrm{LE}}$, los docentes son profesionales de la comunicación y la interiorización de nuevas pautas de comunicación intercultural permite la socialización de las personas; es por eso que aprender a comunicarse con el otro es aprender a interpretar su mundo, a conocer los signos de la sociedad a la que pertenece. De aquí el planteamiento de que el objetivo general de todo proceso de enseñanza-aprendizaje de una lengua extranjera debe estar dirigido a la comunicación intercultural.

Lo expresado hasta aquí permite comprender que la interdependencia tan estrecha entre comunicación y cultura llega al punto de no poder distinguirlas desde una perspectiva pragmática, pues la forma como el hombre se comunica expresa cómo vive.

Por este motivo, desarrollar la competencia comunicativa no se reduce al mero reconocimiento de los elementos lingüísticos; se trata de comprender y de construir nuevos sentidos de vida, lo que implica madurar como personas e integrarse, si así se decide, al contexto sociocultural.

Sin embargo, el encuentro intercultural no siempre es fácil, ni se produce por arte de magia, "más bien al contrario, en un mayor o menor grado, ese encuentro con frecuencia puede convertirse en un "des-encuentro" (Martín Morillas 3), por tanto, la educación para la interculturalidad ha de trabajar sobre las formas de creer, pensar, sentir, comunicarse y actuar, que inciden positiva o negativamente en el encuentro. Durante el aprendizaje de una lengua, tanto la propia como la extranjera, se pone muy claramente en juego esa relación de transferencia entre comunicación, valoración y educación; quiere ello decir, que aprender una lengua fuera del país de origen no es solo aprender su gramática, su pronunciación o su léxico, también implica una lección continua de interculturalidad. Es aprender otra manera de ver el mundo, de acercarse a él, de comportarse con los demás, de saber qué características tiene esa cultura. Desde el punto de vista educativo, y por usar un símil, puede decirse que en los cursos de E LLE se "cuece" mucha interculturalidad, y que en esta cocina (aula) pueden prepararse "platos" (actividades interculturales) interesantes y motivadores que a la par alimenten (informen y eduquen) y propicien el encuentro intercultural. En ese entorno, el profesor puede ayudar a anticipar, pronosticar y desviar las interferencias culturales que avivan los desencuentros. Obviamente, eso quiere decir, según Martín Morrillas:

[...] que la clase de idiomas puede ser el lugar ideal donde se estudie y se reflexione sobre las diferencias y similitudes en cuanto a creencias, valores, actitudes, etc. relativas a los hablantes de otras lenguas - culturas a fin de aprender sobre "ellos" y sobre "nosotros", sobre sus identidades y nuestras identidades, incluyendo perspectivas, valoraciones, formas de pensar, de ser, de actuar, etc. (58)

El estudiante en nuestras aulas adquiere una conciencia intercultural si conoce, percibe y comprende que, entre su mundo de origen y el de las otras personas presentes en el aula, existen similitudes y diferencias distintivas, se enriquece con esa serie de culturas, más amplias de la que conlleva la lengua materna y la lengua que estudia. Solo así adquiere destrezas interculturales que incluyen la capacidad de relacionar culturas, la sensibilidad cultural, la capacidad de mediación y la de superar los estereotipos.

\section{Las tres etapas para el logro de la sensibilidad intercultural}

Existen varias clasificaciones que caracterizan los diferentes grados de desarrollo de la competencia intercultural. Bennett (citado en Torres Beltrán 73) propone un Modelo desarrollador de la sensibilidad intercultural que hemos seleccionado por varias razones: se refiere a la competencia intercultural general, en el sentido de que describe cómo los individuos pueden ir del etnocentrismo, pasando por varias etapas, hasta llegar a despertar su sensibilidad (entendida como la facultad de sentir vivamente por otras culturas); asume que existen características de cada una de las etapas que tienen que ser superadas para pasar a las características más complejas de las etapas subsiguientes; y se basa en dos tipos de investigaciones: una, en la observación de la forma en que diferentes grupos humanos enfrentan la nueva experiencia cultural, y otra conceptual, apoyada en teorías del constructivismo que plantean que gran parte de la experiencia de la realidad se produce mediante constructos o categorías complejas que se integran para formar el mapa mental. 
De esta forma tendremos tres etapas globales:

1. La etapa etnocéntrica, en la cual el individuo considera su propia cultura como la verdadera interpretación de la realidad y por tanto existe un rechazo hacia la idea de la diferencia cultural, a la que perciben como una amenaza implícita o explícita a su propia experiencia cultural.

2. La etapa etnorrelativa, en la cual el sujeto reconoce conscientemente que todo comportamiento, incluyendo el propio, ocurre en un contexto cultural y percibe las restricciones que esto impone a su propia experiencia. Por esta razón está abierto a las diferencias culturales, como forma de enriquecimiento de su experiencia de la realidad y como vía para comprender a personas de otras culturas. A esta actitud, en apariencia respetuosa y tolerante, le falta la búsqueda del encuentro.

3. La etapa intercultural, en la cual la persona, partiendo del respeto, supera las carencias del relativismo cultural. Añade el respeto por las otras culturas y la búsqueda de un encuentro en situación de sinergia y de igualdad. Tiene una visión crítica de las culturas, en la que se acepta, pero puede rechazar alguna de sus instituciones.

Con frecuencia, en los análisis de textos o en las interacciones, los estudiantes evidencian algunos indicios de etnocentrismo que se manifiestan por medio de actitudes sutiles, posiciones nacionalistas, cerradas, poco dispuestas al diálogo o de marcas discursivas que expresan predisposición hacia el otro, y que pueden ir desde determinada entonación, hasta el uso del silencio para evitar el tratamiento de algunos temas; se observa, además, el empleo de frases con semántica valorativa axiológicamente negativa:

1. Da muestras aparentes de compasión y de altruismo.

2. Usa la hipérbole.

3. Hace comparación negativa.

4. Presenta generalizaciones.

Estos indicios generan conflictos, incomprensiones, prejuicios e irrespeto a la cultura "del otro". En consecuencia, es necesario desarrollar en los estudiantes la capacidad de aceptar, de dialogar, de respetar los valores, las ideas, los puntos de vista y las opiniones de los demás, en condiciones de interculturalidad.
El modelo de Bennett concibe al etnocentrismo como una tendencia humana natural, de la cual es posible transitar progresivamente hacia etapas superiores de sensibilidad cultural. Ha sido pedagógicamente estructurado y articulado con el aprendizaje de lenguas extranjeras, a fin de facilitar el aprendizaje cultural a partir de la interacción entre los estudiantes, con la guía del profesor como mediador de esta interacción. Por tanto, el aprendizaje está basado en la discusión, conceptualización, aclaración, clarificación, y resumen de las experiencias facilitadas por el proceso de aprendizaje y por sus propias vivencias.

\section{El tratamiento de los valores para favorecer el desarrollo de la competencia intercultural}

Pese a que los estudios sobre esta temática son aún bastante recientes, no son pocos los investigadores que en Europa y en América han trabajado en ella, aunque con objetivos diferentes: en muchos casos, tratar el tema migratorio y, en otros, ampliar el horizonte apreciativo de los estudiantes que adquieren una segunda lengua.

Este artículo constituye el antecedente de un diseño linguodidáctico para la solución de malentendidos, estereotipos y conflictos interculturales que se manifiestan en el proceso de aprendizaje de una LE, en el que participen estudiantes de diferentes culturas, puesto que en ella se expresan valores universales a partir del conocimiento de la propia cultura y de la comprensión de las otras.

La problemática de los valores es objeto de discusión, de análisis, y de reflexión en foros y eventos nacionales e internacionales. Al enfocar su estudio, se debe tener presente que los valores existen en la realidad social y, según Fabelo Corzo (51), "mediatizan la percepción que nos formamos de los demás y de nosotros; establecen las bases para juzgar a los otros y al propio sujeto, así como sus acciones; y también justifican todo tipo de influencia que se pretenda ejercer en la relación con los demás".

Esto quiere decir que los valores se desarrollan en una persona mediante el proceso de socialización y están en correspondencia con el sistema social en que el sujeto se desenvuelve.

Para el ser humano siempre han existido entidades valiosas: el bien, la verdad, la belleza, la felicidad, la virtud. Sin embargo, el criterio para darles valor puede variar. Es posible valorar de acuerdo con las regiones geográficas o culturas, criterios estéticos, esquemas 
sociales, costumbres, principios éticos, etc. Estos son producto de cambios y transformaciones a lo largo de la historia. Surgen con un significado especial y cambian o desaparecen en las distintas épocas con arreglo a las condiciones históricas donde ellos surgen y se desarrollan: lo que hoy aquí es valioso, mañana, u hoy mismo, allá, puede no serlo, a causa de un cambio en la relación funcional del objeto en cuestión con lo genéricamente humano.

La pedagoga Esther Báxter (194) hace juicios acertados acerca de la relación sujeto-objeto, al plantear que de dicha relación surge el valor, pero no se reduce a la función de las variables mencionadas, sino que su génesis, expresión y desarrollo tienen lugar en las relaciones sociales, en los vínculos interculturales que se establecen, en la individualidad, como complejas unidades funcionales cognitivo-afectivas que forman parte de la realidad social, se hacen sentir en las enunciaciones y producen la regulación en la actuación del individuo.

El sistema valoral ${ }^{1}$ del individuo es suficientemente estable para reflejar la condición de ser uno mismo y la continuidad de una única personalidad socializada dentro de una cultura y de una sociedad determinada que es caracterizada, precisamente, por la pervivencia valoral. Sin embargo, según estos mismos análisis, los procesos madurativos, las relaciones con personas de contexto más amplios o diferentes, las oposiciones axiológicas que a veces surgen en la comunicación provocan en el sujeto conflictos que lo obligan a decidir en sus preferencias, todo lo cual puede provocar la pérdida de algunos valores o una reordenación del sistema valoral. De ahí que el valor y el sistema de valores, a pesar de sus características de perdurabilidad, sean lo suficientemente flexibles para permitir recolocaciones de las prioridades preferibles, como resultado de los aportes en la cultura, los cambios en la sociedad y en la experiencia personal.

La incomprensión, los malentendidos, los conflictos culturales, las manifestaciones de estereotipos y las actitudes negativas pueden ser frecuentes en grupos multiculturales formados por personas adultas, que participan en los procesos de aprendizaje de una LE fuera de su país de origen. Se hace necesario, entonces, reflexionar acerca de los valores éticos, de interacción social e identitarios que se evidencian en una clase multicultural en la que están representadas culturas disímiles.

Desde de la perspectiva de la clase de $\mathrm{E} \backslash \mathrm{LE}$, es importante que el ordenamiento y el reordenamiento de

1 Adjetivo relativo a los valores, según Escámez Sánchez y Ortega Ruiz, en La enseñanza de actitudes y valores (1995). valores se realice en contextos cooperativos, porque un proceso de construcción de nuevos significados y sentidos de vida, como el que se genera mediante el aprendizaje de una LE, supone la definición de posiciones valorativas, orientaciones y puntos de vistas que pueden llegar a construir un referente común solo a partir del diálogo reflexivo en el campo de los valores. Cuando las personas argumentan y brindan sustentaciones para sus puntos de vista, necesitan escuchar a los demás y hacer interconexiones entre lo que es suyo y lo que es "del otro", y puede asegurarse, en definitiva, que van construyendo el conocimiento junto a él. Para ello, comparan, reconocen lo valioso en el otro, hacen inferencias y elaboran alternativas, es decir, recrean la realidad. En ese aprendizaje permanente y desarrollador se capacitan para ejercer sus roles sociales de manera más integral, constructiva y solidaria, como ciudadanos que seleccionan entre todo lo existente en el Universo, aquello que les resulta valioso y construyen, junto a la otra persona, de manera creativa, sus propios sentidos de vida.

Como lo indica su nombre, los Cursos de Corta Duración (CCD) son extremadamente breves ( 80 horas), por lo que no sería posible el tratamiento de una amplia gama de valores. Se considera más aconsejable abordar aquellos de connotación ética, de interacción social e identitaria, por cuanto se ajustan mejor a la clase multicultural y pueden contribuir a la competencia intercultural.

En la obra Valores esenciales para la vida en familia y en comunidad, Bello Porras (45-83) relaciona una serie axiológica de valores, de los cuales he hecho esta selección y clasificación para trabajarlos en el repertorio de textos que se propone, pues dada la duración del curso, resulta del todo imposible tratar todos los valores:

Tabla 2. Selección de valores clasificados en tres categorías

\begin{tabular}{|l|l|l|}
\hline \multicolumn{1}{|c|}{ Valores éticos } & \multicolumn{1}{c|}{$\begin{array}{c}\text { Valores de } \\
\text { interacción social }\end{array}$} & Valores identitarios \\
\hline La honestidad & El amor & La comunicación \\
\hline La discreción & El cooperativismo & La valentía \\
\hline La sinceridad & La bondad & La alegría \\
\hline La modestia & La convivencia & La pasión \\
\hline El respeto & La aceptación & La solidaridad \\
\hline
\end{tabular}

Fuente. Elaboración propia.

Tratar estos aspectos axiológicos no significa formar, ni inculcar, ni desarrollar valores en los estudiantes que acceden a los cursos de E/LE en la UH, sino 
facilitar que los participantes desarrollen y consoliden una competencia comunicativa intercultural, en un clima distendido, interaccionando, explicitando los valores sociales e identitarios que se encuentran en los diferentes materiales textuales y en los análisis que realizan en las actividades de compresión lectora y de interacción y expresión oral. No debe perderse de vista el ambiente de reconocimiento a la cultura del otro, de diálogo abierto, de respeto, de comprensión, de negociación y de tolerancia.

\section{Metodología}

La metodología empleada en el desarrollo de esta investigación reúne facetas de información teórica documental, así como otras de carácter más práctico que detallaremos en este mismo apartado.

La revisión bibliográfica se centra fundamentalmente en documentación de carácter teórico y reúne los trabajos más importantes sobre Teoría de la comunicación, así como cuestiones fundamentales de la Didáctica de lenguas extranjeras y, no menos importante, trabajos dedicados al tratamiento de la interculturalidad, tanto en lo referido al diálogo intercultural propiamente dicho como a su aplicabilidad en procesos didácticos. Tenemos presente aquí que los trabajos desarrollados hasta el momento sobre cuestiones axiológicas se refieren mayoritariamente a lo que ha dado en llamarse Educación en valores, destinados a la etapa escolar, o bien a la integración de inmigrantes en entornos o zonas geográficas receptoras.

El diseño didáctico propiamente dicho estuvo combinado con el ensayo experimental de las propuestas. Esta faceta incluyó la evaluación regular de los resultados parciales de la experimentación, según el modelo de investigación en la acción que es propio de las disciplinas didácticas. Es conveniente resaltar que los CCD, hacia los cuales va dirigido la investigación y que son el objeto de la experimentación, tienen una duración global de cuatro semanas y que la finalización de cada uno de ellos conecta con la puesta en marcha de una nueva edición.

Una de las cuestiones más determinantes de la experimentación fue la selección de textos (y otros recursos) que sirven como base al desarrollo de las clases de nivel superior. Esta selección se llevó a cabo a partir de los siguientes criterios:
1. Textos literarios ( 12 hasta el momento).

2. Las temáticas debían estar relacionadas con valores éticos, de interacción social o identitarios (de la identidad cubana y latinoamericana).

3. Debían ofrecer amplias posibilidades de explotación didáctica.

4. Los textos debían ser breves (desde media, hasta una cuartilla y media).

La efectividad de este catálogo se ha comprobado y validado también por medio de la observación y del funcionamiento en las clases de otros docentes.

Las evaluaciones sistemáticas (mes a mes) de la progresión o adquisición de la competencia intercultural y de la sensibilidad valoral se basaron en mediciones concretas del rendimiento individual de los estudiantes de cada grupo, así como en las observaciones directas sobre la eficacia de las propuestas didácticas, incluyendo aquí la eficiencia de los textos utilizados y de las actividades propuestas para su tratamiento.

\section{Resultados}

Este artículo parte de una investigación en curso que propone un repertorio de textos cuyas actividades propician el tratamiento de determinados valores éticos, de interacción social e identitarios, que favorecen el progresivo desarrollo de la competencia intercultural, así como las declaraciones axiológicas de los estudiantes basadas en un clima distendido, el respeto a la cultura "del otro", el diálogo franco y abierto, la cooperación, la tolerancia y otros factores.

$\mathrm{Al}$ elaborar y aplicar la propuesta, las evaluaciones sistemáticas realizadas, así como las observaciones directas sobre la eficacia de las propuestas didácticas, incluyendo aquí el repertorio de textos utilizados y las actividades planteadas para su tratamiento, se demuestra no solo el nivel de satisfacción de los aprendices, sino la ampliación de la capacidad de diálogo, de entendimiento y de comprensión, lo cual conduce indiscutiblemente a la clarificación de valores y al logro de la competencia comunicativa intercultural, lo cual constituye el objetivo principal de la investigación.

Después de once meses de experimentación, en los que se aplicaron todos los textos del repertorio, que incluyeron poemas de José Martí, Mario Benedetti 
y Jesús Orta (El Indio Naborí), así como cuentos del propio Martí, de Gabriel García Márquez, de Onelio Jorge Cardoso, de Eduardo Galeano y de otros quizás menos conocidos, los resultados arrojan que el tratamiento de valores universales en grupos multiculturales de nivel superior que acceden a los cursos de E/LE, en la Universidad de La Habana, agrupados por su nivel de lengua, pero con disímiles estilos de aprendizaje, edades, creencias y procedentes de culturas diversas, y por eso, portadores de sistemas de valores distintos, pueden mediante el estudio y el análisis de textos conocer, reconocer y ampliar sus conocimientos lingüísticos, pero, además, pueden desarrollar y consolidar una competencia comunicativa intercultural de nivel superior, precisamente mediante las actividades de comprensión lectora y la explicitación de sus propios valores culturales.

\section{Conclusiones}

La coyuntura internacional de los tiempos actuales, dominada por el desarrollo acelerado de la ciencia, de la informática y de las comunicaciones, demanda acciones pedagógicas que garanticen la formación de hombres plenos, capaces de dominar las nuevas tecnologías para darles un uso apropiado, correcto y enriquecedor.

Gran parte de los aprendices, al llegar a los cursos de E/LE de la UH, se encuentran en la etapa etnorrelativa, puesto que han entrado, anteriormente, en relación de intercambio con otras culturas, pero este no es el caso de todos, por lo que es necesario que en los cursos de aprendizaje de lenguas se realice un trabajo de sensibilización mediante un proceso de cooperación de profesores y estudiantes, para que aquellos que no han llegado a la conciencia intercultural puedan alcanzarla $y$, en sentido general, todos enriquezcan su espectro valoral a partir de sus vivencias en el país donde estudian la lengua, y de los análisis, debates, diálogos y actividades grupales propias de la clase en la que manifiestan diferentes opiniones y perspectivas.

La conversación sobre valores en el aula depende en gran medida de que el estímulo, mayoritariamente, de actividades basadas en textos escritos aborden directamente cuestiones axiológicas universales y propicien la interacción oral en el proceso didáctico.

Abordar el tratamiento de valores en la clase $\mathrm{E} / \mathrm{LE}$ no equivale a defender como válido el sistema de valores propio de la cultura meta, por más que sea esta la que más se vea reflejada en la conducta verbal del escenario donde se aprende. Se trata de que los participan- tes reconozcan, comprendan y respeten, en el proceso de enseñanza-aprendizaje, la diversidad cultural, no con el fin de abrazar o rechazar conscientemente una u otra posición, sino para que dichas diferencias no dificulten u obstaculicen los procesos comunicativos, garantes del desarrollo de la competencia comunicativa intercultural.

\section{Referencias}

Aguado Odine, Teresa. “Pedagogía intercultural.” McGraw-Hill/ Interamericana de España. (2003). 5 oct. 2012. Web. http:// www.casadellibro.com/libro-pedagogia-intercultural/

Báxter Pérez, Esther. La educación en valores: Papel de la escuela. La Habana: Editorial Pueblo y educación, 2003. Impreso.

Bello Porras, José Gregorio. “Cultivo de los valores.” Valores esenciales para la vida en familia y en comunidad. Caracas: Consejo Nacional de Cultura, 2004. Impreso.

Diviñó González, Esther. El desarrollo de la sensibilidad y el enriquecimiento valoral a través de las actividades discursivas orales en el nivel elemental alto del aula intercultural de español como lengua extranjera, en los cursos de corta duración. Tesis. Universidad de La Habana, 2008. Impreso.

Estrategia linguodidáctica para el tratamiento de los valores, a partir de la comprensión lectora en condiciones de interculturalidad, 2012. Tesis. Universidad de La Habana. En desarrollo.

Escámez Sánchez, Juan y Ortega Ruiz, Pedro. La enseñanza de actitudes y valores. Valencia, España: Editorial NAU Libres, 1995. Impreso.

Fabelo Corzo, José Ramón. Los valores y sus desafíos actuales. La Habana: Editorial José Martí, 2003. Impreso.

Kramsch, Claire. Contexto y cultura en la enseñanza de lenguas. Oxford: University, 1996. Impreso.

Llanes Barbuzano, Rómula. Modelo didáctico para el tratamiento de la valoración apreciativa ético-psicológica en la clase de lengua extranjera. Tesis. Universidad de La Habana, 2004. Impreso.

Martín Morillas, José Manuel. "La enseñanza de la lengua: un instrumento de unión entre culturas". (2000). 2 feb. 2007. Web. http://www.ub.edu/filhis/culturele/morillas.html

Sánchez Pérez, Aquilino. "Lengua y cultura". La enseñanza de idiomas en los últimos cien años: métodos y enfoques. Madrid, España: Sociedad General Española de Librerías, 2009. Impreso.

Serrano Olmedo, Rocío. "Guía de conocimiento sobre educación intercultural”. (2011). 22 jul. 2012. Web. http:// www.gloobal.net/iepala/gloobal/fichas/ficha.php?entid $\mathrm{ad}=$ Textos\&id=5336\&opcion=documento 
Torres Beltrán, Hilda. El desarrollo de la competencia comunicativa intercultural de los estudiantes de la licenciatura en inglés como segunda lengua extranjera a través del curso panorama histórico-cultural de los EEUU. Tesis. Universidad de La Habana, 2003. Impreso.
Trujillo Sáez, Fernando. "En torno a la interculturalidad: reflexiones sobre cultura y comunicación para la didáctica de la lengua”. Portalingual. Abril del 2002: 11-15. Impreso.

Zayas Martínez, Francisco. "La literatura en la enseñanza del alemán como segunda lengua extranjera." Tesis. Universidad de Cádiz, España, 2002. Impreso. 